\title{
A FACTORIZATION OF CONDITIONAL EXPECTATIONS ON KAC ALGEBRAS AND QUANTUM DOUBLE COSET HYPERGROUPS*
}

\section{ФАКТОРИЗАЩЯ УМОВНИХ СПОДПВАНЬ НА АЛПЕБРАХ КАЩА I КВАНТОВІ ГПIЕРГРУПИ, ЩО ПОВ'ЯЗАНІ З ПОДВІЙНИИМИ КЛАСАМИ СУМІЖНОСТІ}

We prove that a conditional on a Kac algebra, under certain conditions, decomposes into a composition of two conditional expectations of a special type and gives rise to a compact quantum hypergroup connected to a quantum Gelfand pair.

Доведепо, що умовие сподівания ша алгебрі Каца, лке задоволыле певні умови, може бути розкладене в компознцію двох умовних сподівань спеціалыного типу і породжуе компактну квантоny rineprpyny, nов' язапу з квагrтового пароьо Гельфанда.

Introduction. Compact quantum hypergroups were introduced in [1] as a structure that simultaneously generalizes the usual hypergroups [2, 3], compact matrix pseudogroups [4], and bialgebras of biinvariant functions associated with quantum Gelfand pairs [5-7].

A compact quantum hypergroup is a unital $C^{*}$-algebra equipped with a coassociative completely positive coproduct that preserves the unit element and satisfies some additional axioms. Examples of compact quantum hypergroups associated with quantum Gelfand pairs were constructed in [1]. In [8], a general construction of quantum hypergroups using conditional expectations on compact quantum groups was proposed. This construction generalizes that for orbital morphisms [9] of the usual DJS-hypergroups and includes the double coset construction for quantum groups $[5,7]$ and an analogue of the Delsart construction for quantum hypergroups. In [8], several series of nontrivial finite-dimensional quantum hypergroups were constructed from nontrivial Kac algebras obtained in $[10,11]$, by twisting the classical series of finite groups.

The examples of compact quantum hypergroups constructed in [8] had the property that the expectation preserved the counit. We call such conditional expectations counital (see Definition 3.1). The conditional expectation that arises in the double coset construction is not of such a type. In this paper, however, we show that a conditional expectation on a Kac algebra, if it satisfies certain cinditions, is a composition of conditional expectations of the above mentioned two types. This means that the two types of examples that were considered make, in a certain sense, building blocks for constructing compact quantum hypergroups from a conditional expectation on a Kac algebra.

The paper is organized as follows. Section 1 contains nesessary preliminaries on compact quantum hypergroups and quantum double cosets. In Section 2, we describe the construction of compact quantum hypergroups using conditional expectations on quantum hypergroups (and, in particular, on a Kac algebra) and give a new example of a compact quantum hypergroup. In Section 3, we prove the main theorem on factorization of a conditional expectation.

1. Preliminaries. 1.1. By $\mathcal{A}=(A, \Delta, \varepsilon, \kappa, \mu)$ we denote a compact $\mathrm{Kac} C^{*}$ algebra [12], $\triangle: A \rightarrow A \otimes A$ is a comutiplication with the $C$-projective tensor square $A \otimes A, \varepsilon: A \rightarrow \mathbb{C}$ is a counit, $\kappa: A \rightarrow A$ is an antipode, and $\mu$ is a Haar measure.

For a Kac algebra $\mathcal{A}$, we denote by $\hat{A}$ the Banach space of continuous

* This work has been partially supported by the Ukrainian committee for fundamental studies (project No 01.07.071). 
functionals on the $C$-algebra $A$, with the norm $\|\xi\|=\sup _{|a|=1}|\xi(a)|$. For $\xi, \eta \in$ $\in \hat{A}$, we define a product - and an involution $\star$ by

$$
(\xi \cdot \eta)(a)=(\xi \otimes \eta) \Delta(a), \quad \xi^{\star}(a)=\bar{\xi}\left(\kappa(a)^{*}\right), \quad a \in A .
$$

With these operations, $\hat{A}$ is an involutive Banach algebra. For $\xi \in \hat{A}$ and $a \in A$, we denote, as usual,

$$
\xi \cdot a=(\mathrm{id} \otimes \xi) \circ \Delta(a), \quad a \cdot \xi=(\xi \otimes \mathrm{id}) \circ \Delta(a) .
$$

By $\hat{A} \otimes \hat{A}$, we understand the completion of the algebraic tensor product with respect to a cross-norm adjoint to the projective cross-norm on $A \otimes A$ [13]. The multiplication in the $C$-algebra $A$ gives rise to a comultiplication $\hat{m} \cdot: \hat{A} \rightarrow \hat{A} \otimes \hat{A}$. Let $\xi \in \hat{A}$ be a state and $\rho: A \rightarrow B\left(H_{\xi}\right)$ be the GNS-representation of $A$ corresponding to the state $\xi, H_{\xi}$ and $B\left(H_{\xi}\right)$ the corresponding Hilbert space and the *-algebra of bounded operators on $H_{\xi}$. Then, for an orthonormal basis $\left\{e_{i}\right\}_{i=1}^{\infty}$ in $H_{\xi}$ with $\xi_{i j}(a)=\left(\rho(a) e_{j}, e_{i}\right)_{H_{\xi}}$ and $e_{i}=1$, so that $\xi_{11}=\xi$, we have

$$
\hat{m}(\xi)=\sum_{i=1}^{\infty} \xi_{1 i} \otimes \xi_{i 1} .
$$

1. 2. A compact quantum hypergroup [1], $B=\left(B, \delta, \varepsilon, \star, \sigma_{t}, \mu\right)$, is a unital $C^{*}$ algebra $B$ with a completely positive coassociative comultiplication $\delta: B \rightarrow B \otimes B$, where $B \otimes B$ is the projective $C^{\star}$-square, a counit $\varepsilon: B \rightarrow \mathbb{C}$, a coinvolution $\star$, a continuous one-parameter group of automorphisms $\sigma_{t}$, and a Haar measure $\mu$. We additionally assume that

$$
\begin{gathered}
(a \cdot b)^{\star}=a^{\star} \cdot b^{\star}, \quad \delta \circ *=(* \otimes *) \circ \delta, \\
\varepsilon(a \cdot b)=\varepsilon(a) \varepsilon(b), \quad \delta(1)=1 \otimes 1, \\
\star \circ *=* \circ \star
\end{gathered}
$$

for all $a, b \in B$ (here $*$ is the involution in $C^{*}$-algebra $B$ ). The conditions imposed on the one-parameter group $\sigma_{t}$ are the following:

(a) there exist dense subalgebras $B_{0} \subset B$ and $\vec{B}_{0} \subset B \otimes B$ such that the oneparameter groups $\sigma_{t}$ and $\sigma_{t} \otimes$ id, id $\otimes \sigma_{t}$ can be extended to complex one-parameter groups $\sigma_{z}$ and $\sigma_{z} \otimes$ id, id $\otimes \sigma_{z}, z \in \mathbb{C}$, of automorphisms of the algebras $B_{0}$ and $\tilde{B}_{0}$ respectively;

(b) $B_{0}$ is invariant with respect to $*$ and $\star$, and $\delta\left(B_{0}\right) \subset \widetilde{B}_{0}$;

(c) the following relations hold on $B_{0}$ for all $z \in \mathbb{C}$ :

$$
\begin{gathered}
\delta \circ \sigma_{z}=\left(\sigma_{z} \otimes \sigma_{z}\right) \circ \delta, \\
\mu\left(\sigma_{z}(a)\right)=\mu(a) ;
\end{gathered}
$$

(d) there exists $z_{0} \in \mathbb{C}$ such that the Haar measure $\mu$ satisfies the following strong invariance condition for all $a, b \in B_{0}$ :

$$
(\mathrm{id} \otimes \mu)\left[\left(\left(* \circ \sigma_{z_{0}} \circ \star \otimes \mathrm{id}\right) \circ \delta(a)\right) \cdot(1 \otimes b)\right]=(\mathrm{id} \otimes \mu)((1 \otimes a) \cdot \delta(b)) .
$$


We finally assume that the Haar measure $\mu$ is faithful on $B_{0}$.

The antipode $\kappa$ is defined by

$$
\kappa=* \circ \sigma_{z_{0}} \circ \star
$$

Then for all $a, b \in B_{0}$,

$$
\begin{gathered}
\kappa(a b)=\kappa(b) \kappa(a), \quad \delta \circ \kappa=\Pi \circ(\kappa \otimes \kappa) \circ \delta, \\
\mu \circ \kappa=\mu, \quad \kappa(1)=1, \quad \varepsilon \circ \kappa=\varepsilon .
\end{gathered}
$$

The antipode is invertible with $\mathrm{K}^{-1}=\star \circ \sigma_{-z_{0}} \circ *$. With these notations, the relation in (d) is

$$
(\mathrm{id} \otimes \mu)((\kappa \otimes \mathrm{id}) \circ \delta(a) \cdot(1 \otimes b))=(\mathrm{id} \otimes \mu)((1 \otimes a) \cdot \delta(b)) .
$$

1.3. Let $\mathcal{A}_{1}=\left(A_{1}, \Delta_{1}, \varepsilon_{1}, \kappa_{1}, \mu_{1}\right)$ and $\mathcal{A}_{2}=\left(A_{2}, \Delta_{2}, \varepsilon_{2}, \kappa_{2}, \mu_{2}\right)$ be two Kac algebras and let $\pi: A_{1} \rightarrow A_{2}$ be a Hopf $C^{*}$-algebra epimorphism, i. e., $\pi$ is a $C^{*}$ algebra epimorphism satisfying

$$
(\pi \otimes \pi) \circ \Delta_{1}=\Delta_{2} \circ \pi, \quad \varepsilon_{2} \circ \pi=\varepsilon_{1} .
$$

Define

$$
\begin{gathered}
A_{1} / A_{2}=\left\{a \in A_{1}:(\text { id } \otimes \pi) \circ \Delta_{1}(a)=a \otimes 1\right\}, \\
A_{2} \backslash A_{1}=\left\{a \in A_{1}:(\pi \otimes \text { id }) \circ \Delta_{1}(a)=1 \otimes a\right\}, \\
A_{2} \backslash A_{1} / A_{2}=A_{2} \backslash A_{1} \cap A_{1} / A_{2} .
\end{gathered}
$$

The Banach space $B=A_{2} \backslash A_{1} / A_{2}$ is an involutive algebra with the unit 1. A comultiplication $\delta: B \rightarrow B \otimes B$ is defined by restricting

$$
\delta=\left(\text { id } \otimes \mu_{2} \circ \pi \otimes \text { id }\right) \circ\left(\Delta_{1} \otimes \text { id }\right) \circ \Delta_{1}
$$

to $B$ and $\star: B \rightarrow B$ is given by

$$
a^{\star}=\kappa_{1}(a)^{*},
$$

where $a \in B$. Set $\varepsilon$ to the restriction of $\varepsilon_{1}$ and $\mu$ the restriction of $\mu_{1}$ to $B$. Then $\mathcal{B}=\left(B, \delta, \varepsilon, \star, \sigma_{t}, \mu\right)$, where $\sigma_{t}=\mathrm{id}$, is a compact quantum hypergroup [1], we denote it, by abuse of notations, by $A_{2} \backslash A_{1} / A_{2}$, and call the quantum double coset of $\mathcal{A}_{1}$ with respect to $\mathcal{A}_{2}$. The map $P: A_{1} \rightarrow B$ defined by

$$
P=\left(\mu_{2} \circ \pi \otimes \mathrm{id} \otimes \mu_{2} \circ \pi\right) \circ\left(\Delta_{1} \otimes \mathrm{id}\right) \circ \Delta_{1}
$$

is the conditional expectation corresponding to the double coset construction. By setting $A_{2}=\mathbb{C} 1$ with trivial operations, a compact quantum pseudogroup $\mathcal{A}_{1}$ can be regarded as a compact quantum hypergroup.

2. Conditional expectations on a Kac algebra. 2.1. Let $\mathcal{A}=\left(A, \Delta, \varepsilon, \star, \sigma_{t}, \mu\right)$ be a compact quantum hypergroup and $\kappa$ the antipod defined by (2). Let $B$ be a unital $C$-subalgebra of $A$ and $P: A \rightarrow B$ the corresponding $\mu$-invariant conditional expectation [14] (i. e., $\mu \circ P=\mu$ ). We will need the following version of Theorem 2.1 in [8]. The conditions of Theorem 2.1 below are given in a form simpler then in [8].

Theorem 2. 1. Let $\mathcal{A}$ be a compact quantum hypergroup, $B$ a unital $C^{*}$ subalgebra of the $C^{*}$-algebra $A$, and $P$ a conditional expectation from $A$ to $B$. Assume that, for the dense subalgebras $A_{0}$ and $\vec{A}_{0}$, we have $P\left(A_{0}\right) \subset A_{0},(P \otimes$ $\otimes \mathrm{id})\left(\tilde{A}_{0}\right) \subset \tilde{A}_{0}$, and (id $\left.\otimes P\right)\left(\tilde{A}_{0}\right) \subset \tilde{A}_{0}$. Let $P$ satisfy the following conditions: 


$$
\begin{gathered}
(P \otimes \text { id }) \Delta \circ P=(P \otimes P) \Delta=(\text { id } \otimes P) \Delta \circ P, \\
P \circ \star=\star \circ P, \\
P \circ \sigma_{z}=\sigma_{z} \circ P, \\
\mu \circ P=\mu .
\end{gathered}
$$

Set, for all $x \in B$,

$$
\tilde{\Delta}(x)=(P \otimes P) \Delta(x) .
$$

Then $\left(B, \tilde{\Delta}, \varepsilon, \star \sigma_{t}, \mu\right)$ is a compact quantum hypergroup.

Proof. It suffices to show that the conditions of this theorem imply the conditions of Theorem 2.1 in [8]. The only fact that we need to prove is that $\varepsilon$ is a counit of coalgebra $(B, \tilde{\Delta})$. But this readily follows from (6):

$$
\begin{aligned}
& (\varepsilon \otimes \mathrm{id}) \tilde{\Delta}(b)=(\varepsilon \otimes \mathrm{id})(P \otimes P) \Delta(b)= \\
& =(\varepsilon \otimes \mathrm{id})(\mathrm{id} \otimes P) \Delta(P b)=P b=b
\end{aligned}
$$

for all $b \in B$.

2.2. Delsart hypergroups. Let $\mathcal{A}=(A, \delta, \varepsilon, \kappa, \mu)$ be a compact matrix pseudogroup [4] and let $\Gamma$ be a compact group of Hopf $C^{*}$-algebra automorphisms of $\mathcal{A}$, i. e., each $\gamma \in \Gamma$ is a $C$-algebra automorphism satisfying $(\gamma \otimes \gamma) \circ \Delta=\Delta \circ \gamma, \varepsilon \circ$ ० $\gamma=\varepsilon$, and also $\gamma\left(A_{0}\right) \subset A_{0}$ with $\gamma \circ \kappa=\kappa \circ \gamma$, where $A_{0}$ is the *-subalgebra of $A$ generated by matrix elements of the fundamental corepresentation. Let $v$ be a Haar measure of $\Gamma$ such that $v(\Gamma)=1$. Denote by $B=\{b \in A \mid \gamma(b)=b\}$ the fixed point algebra for the $\Gamma$-action. Define a mapping $P: A \rightarrow B$ by $P a=\int_{\Gamma} \gamma(a) d \gamma$. It was proved in [8] that $P$ is a conditional expectation on $A$ satisfying hypotheses of Theorem 2.1 and $\mathcal{B}=\left(B, \widetilde{\Delta}, \varepsilon, \star, \sigma_{t}, \mu\right)$ is a compact quantum hypergroup, which we call a quantum Delsart hypergroup. Here $\sigma_{t}$ is defined in terms of the family of modular homomorphisms on the compact matrix pseudogroup, see [1]. Also, for all $b$ $\in B$, the following formula for the comultiplication $\tilde{\Delta}$ holds:

$$
\tilde{\Delta}(b)=(\mathrm{id} \otimes P)(\Delta(b)) \text {. }
$$

Examples of finite dimensional quantum Delsart hypergroups were given in [8]. Here we give an example of a compact quantum Delsart hypergroup associated with the compact matrix pseudogroup $S U_{q}(2)$.

2.3. Example. The $C^{*}$-algebra of the quantum group $S U_{q}(2)$ is generated by elements $a, b, c, d$, where $a^{*}=d, b^{*}=-q c$, that satisfy the following relations:

$$
\begin{gathered}
a b=q b a, \quad a c=q c a, \quad b c=c b, \\
b d=q d b, \quad c d=q d c, \quad D_{q}=a d-q b c=1 .
\end{gathered}
$$

The comultiplication and the antipode are defined by the formulas

$$
\begin{array}{cl}
\Delta(a)=a \otimes a+b \otimes c, & \Delta(b)=a \otimes b+b \otimes d, \\
\Delta(c)=c \otimes a+d \otimes c, & \Delta(d)=c \otimes b+d \otimes d, \\
\kappa(a)=d, \quad \kappa(b)=-q^{-1} b, & \kappa(c)=-q c, \quad \kappa(d)=a .
\end{array}
$$

Also, $\varepsilon(a)=\varepsilon(d)=1, \varepsilon(b)=\varepsilon(c)=0$.

For each $\beta \in \mathbb{C},|\beta|=1$, we define $\gamma_{\beta}(a)=a, \gamma_{\beta}(b)=\beta b, \gamma_{\beta}(c)=\beta^{-1} c$, 
$\gamma_{\beta}(d)=d$. Then $\gamma_{\beta}$ is a Hopf $C^{*}$-algebra automorphism of $S U_{q}(2)$. Each primitive root $\beta$ of 1 generates the subgroup $\mathbb{Z}_{n}$ of the group of Hopf $C^{*}$-algebra automorphisms of $S U_{q}(2)$. The corresponding Delsart quantum hypergroup $B$ is nontrivial (i.e., the $\vec{C}$-algebra $B$ is noncommutative and the comultiplication is nonsymmetric) and generated by the elements $a, d$ and $b^{n}, b c, c^{n}$. Also there exists a nontrivial quantum Delsart hypergroup associated with the group $\mathbb{T}$ of the rotation of the circle generated by all $\gamma_{\beta},|\beta|=1$. This hypergroup is generated by the elements $a, b c, d$.

3. Factorization theorem. Let $\mathcal{A}$ be a compact Kac $C^{*}$-algebra, $B$ a subalgebra of $A$, and $1 \in B$. Let $P: A \rightarrow B$ be a conditional expectation satisfying conditions in Theorem 2.1. Denote $\vec{\varepsilon}=\varepsilon \circ P$.

Definition 3.1. A conditional expectation $P$ on a compact quantum hypergroup is called counital if $\varepsilon \circ P=\varepsilon$.

Lemma 3.1. The mapping $\tilde{\varepsilon}: A \rightarrow \mathbb{C}$ is a state on the $C^{*}$-algebra $A$. Regarded as an element of the algebra $\hat{A}, \tilde{\varepsilon}$ is an idempotent, that is, $\vec{\varepsilon}^{2}=\tilde{\varepsilon}$.

Proof. The first claim follows immediately, since $P$ preserves the cone of positive elements of the $C^{*}$-algebra $A, \varepsilon$ is a homomorphism, and $\tilde{\varepsilon}(1)=1$.

To prove the second claim, we have

$$
\begin{aligned}
\tilde{\varepsilon}^{2} & =(\varepsilon \circ P \otimes \varepsilon \circ P) \circ \Delta=(\varepsilon \otimes \text { id }) \circ(\text { id } \otimes \varepsilon \circ P) \circ \Delta \circ P= \\
& =(\text { id } \otimes \varepsilon \circ P) \circ(\varepsilon \otimes \text { id }) \circ \Delta \circ P=\varepsilon \circ P \circ P=\tilde{\varepsilon} .
\end{aligned}
$$

Lemma 3.2. Let $\tilde{\varepsilon}$ be a trace on $A$. The left ideal

$$
J=\left\{a \in A \mid \tilde{\varepsilon}\left(a^{*} a\right)=0\right\}
$$

is a two-sided closed ideal and a coideal in $A$, that is,

$$
\Delta(J) \subset J \otimes A+A \otimes J .
$$

The ideal $J$ is invariant with respect to the involution and coinvolution,

$$
J^{*}=J, \quad J^{\star}=J .
$$

Proof. Since $\widetilde{\varepsilon}$ is a trace, it is immediate that the left ideal $J$ is, in fact, a twosided ideal. To see that $J$ is a coideal, let $a \in J$ and consider

$$
\begin{gathered}
(\tilde{\varepsilon} \otimes \tilde{\varepsilon})\left(\Delta(a)^{*} \Delta(a)\right)=(\tilde{\varepsilon} \otimes \tilde{\varepsilon})\left(\Delta\left(a^{*} a\right)\right)= \\
=\tilde{\varepsilon}^{2}\left(a^{*} a\right)=\tilde{\varepsilon}\left(a^{*} a\right)=0 .
\end{gathered}
$$

Again, using that $\tilde{\varepsilon}$ is a trace,

$$
\tilde{\varepsilon}\left(\left(a^{*}\right)^{*} a^{*}\right)=\tilde{\varepsilon}\left(a a^{*}\right)=\tilde{\varepsilon}\left(a^{*} a\right)=0
$$

for $a \in J$.

Finally, since the involution and the coinvolution commute and $\vec{\varepsilon} \circ \star=\tilde{\varepsilon}$, using (1) we have

$$
\tilde{\varepsilon}\left(\left(a^{\star}\right)^{*} a^{\star}\right)=\tilde{\varepsilon}\left(\left(a^{*} a\right)^{\star}\right)=\tilde{\varepsilon}\left(a^{*} a\right)=0 .
$$

In what follows we assume that $\tilde{\varepsilon}$ is a tracial state on the $C^{*}$-algebra $A$. 
Lemma 3.3. Denote by

$$
\hat{J}=\{\xi \in \hat{A} \mid \xi(a)=0 \text { for all } a \in J\}
$$

the annihilator of $J$ in $\hat{A}$. Then

(i) the state $\tilde{\varepsilon}$ belongs to $\hat{J}$;

(ii) the counit $\varepsilon \in \hat{J}$;

(iii) $\Delta \hat{J} \subset \hat{J} \otimes \hat{J}$;

(iv) $\hat{J}$ is a closed involutive subalgebra of the involutive Banach algebra $\hat{A}$;

(v) $\hat{J}$ is a closed with respect to the map *, where $X^{*}(a)=\bar{X}\left(a^{*}\right)$ for $X \in \hat{A}, a \in A$.

Proof. Since

$$
|\tilde{\varepsilon}(a)|^{2} \leq \tilde{\varepsilon}(1) \tilde{\varepsilon}\left(a^{*} a\right)=0
$$

for all $a \in J$, we obtain that $\tilde{\varepsilon} \in \hat{J}$.

Let us show (ii). Since $\Delta J \subset A \otimes J+J \otimes A$, we have, for any $a \in J$, that

$$
\varepsilon(a)=\tilde{\varepsilon}(\varepsilon(a) 1)=\langle\tilde{\varepsilon}, m((\kappa \otimes \text { id }) \Delta a)\rangle=0 .
$$

Here we denote by $m$ the multiplication in $A$.

To prove (iii), we let $a \in J, b \in A$, and $\xi \in \hat{J}$. Then

$$
\langle\Delta \xi, a \otimes b\rangle=\xi(a b)=0,
$$

since $J$ is a left ideal, whence $\Delta \xi \subset \hat{J} \otimes \hat{A}$. Since $J$ is also a right ideal, we have

$$
\langle\Delta \xi, b \otimes a\rangle=\xi(b a)=0,
$$

whence $\Delta \xi \subset \hat{A} \otimes \hat{J}$. Hence $\Delta \hat{J} \subset \hat{J} \otimes \hat{J}$.

Statements (iv) and (v) follow from the fact that $J$ is a coideal invariant with respect to $*$ and $\star$.

The lemma is proved.

Denote by

$$
A / \hat{J}=\{a \in A \mid a, \xi=\langle\xi, 1\rangle a \text { for all } \xi \in \hat{J}\}
$$

the space of right $\hat{J}$-invariant elements of $A$, and by

$$
\hat{J} \backslash A=\{a \in A \mid \xi, a=\langle\xi, 1\rangle a \text { for all } \xi \in \hat{J}\}
$$

the space of left $\hat{J}$-invariant elements of $A$. Let

$$
\hat{J} \backslash A / \hat{\jmath}=\hat{J} \backslash A \cap A / \hat{J}
$$

be the space of biinvariant elements of $A$.

Lemma 3.4. The space $\hat{\jmath} \backslash A / \hat{J}$ of $\hat{J}$-biinvariant elements is a $C^{*}$ subalgebra of the $C^{*}$-algebra $A$.

Proof. Let us show that $\hat{\jmath} / A$ is a subalgebra of $A$. Let $a, b \in A / \hat{J}$. Since $\Delta \xi \in \hat{J} \otimes \hat{J}$ for all $\xi \in \hat{J}$, we have

$$
\begin{gathered}
a b \cdot \xi=(\xi \otimes \mathrm{id}) \Delta(a b)=(\xi \otimes \mathrm{id}) \Delta(a) \Delta(b)= \\
=(\xi \otimes \mathrm{id})\left(\sum_{i}\left(a_{1}^{i} \otimes a_{2}^{i}\right)\left(b_{1}^{i} \otimes b_{2}^{i}\right)\right)=\sum_{i} \xi\left(a_{1}^{i} b_{1}^{i}\right) a_{2}^{i} b_{2}^{i}=
\end{gathered}
$$




$$
\begin{aligned}
& =\sum_{i}\left\langle\Delta \xi, a_{1}^{i} \otimes b_{1}^{i}\right\rangle a_{2}^{i} b_{2}^{i}=\sum_{i j} \xi_{j}^{1}\left(a_{1}^{i}\right) a_{2}^{i} \xi_{j}^{2}\left(b_{1}^{i}\right) b_{2}^{i}= \\
& =\sum_{j}\left(\xi_{j}^{1} \otimes \mathrm{id}\right) \Delta a\left(\xi_{j}^{2} \otimes \mathrm{id}\right) \Delta b=\sum_{j} a \cdot \xi_{j}^{1} b \cdot \xi_{j}^{2}= \\
& =\sum_{j}\left\langle\xi_{j}^{1}, 1\right\rangle a\left\langle\xi_{j}^{2}, 1\right\rangle b=\left\langle\Delta \xi_{,}, 1 \otimes 1\right\rangle a b=\langle\xi, 1\rangle a b,
\end{aligned}
$$

whence $a, b \in A / \hat{J}$, and $A / \hat{J}$ is a subalgebra.

Since

$$
\begin{aligned}
(\xi \otimes \mathrm{id}) \Delta\left(a^{*}\right) & =(\xi \circ * \otimes *) \Delta(a)=\left(\left(\xi^{*} \otimes \mathrm{id}\right) \Delta(a)\right)^{*}= \\
& =\overline{\left\langle\xi^{*}, 1\right\rangle} a^{*}=\langle\xi, 1\rangle a^{*},
\end{aligned}
$$

$A / \hat{J}$ is an involutive subalgebra. It is clearly norm-closed, hence a $C^{*}$-subalgebra.

All of the above hold for $\hat{J} \backslash A$, whence the claim.

The lemma is proved.

Denote by $C=A / J$ the factor-algebra of the $C^{*}$-algebra $A$ with respect to the ideal $J$. Let $\pi: A \rightarrow C$ be the corresponding morphism of the $C^{*}$-algebras. We can endow the algebra $C$ with a coproduct, $\Delta_{1}(\pi(a))=(\pi \otimes \pi) \Delta a$, a counit $\varepsilon_{1}(\pi(a))=$ $=\varepsilon(a)$, and an antipode $\kappa_{1}(\pi(a))=\kappa(a), a \in A$ (nopte that $\varepsilon_{1}$ is correctly defined since $\varepsilon \in \hat{J}$ ). Then the $C^{*}$-algebra $C$ with the comultiplication $\Delta_{1}$, counit $\varepsilon_{1}$, and the antipode $\kappa_{1}$ is a compact Kac algebra and $\pi: A \rightarrow C$ is a Kac algebra epimorphism.

Lemma 3.5. Define $\pi^{R}(a)=a \cdot \tilde{\varepsilon}$ and $\pi^{L}(a)=\tilde{\varepsilon} . a, a \in A$. Then

(i) $\pi^{L}, \pi^{R}$ are commuting projection on $A$;

(ii) $\pi^{L}$ and $\pi^{R}$ are conditional expectations on $A$;

(iii) for any $\xi \in \hat{J}$ the following equalities hold:

$$
\begin{aligned}
& \xi . \tilde{\varepsilon}=\langle\xi, 1\rangle \tilde{\varepsilon}, \\
& \tilde{\varepsilon} \cdot \xi=\langle\xi, 1\rangle \tilde{\varepsilon} ;
\end{aligned}
$$

(iv) $\pi^{R}(A)=A / \hat{J}$ and $\pi^{L}(A)=\hat{J} \backslash A$.

Proof. For $a \in A$, we have

$$
\left(\pi^{L}\right)^{2}(a)=\tilde{\varepsilon} \cdot(\tilde{\varepsilon} \cdot a)=\tilde{\varepsilon}^{2} \cdot a=\pi^{L}(a),
$$

since $\tilde{\varepsilon}^{2}=\tilde{\varepsilon}$. Therefore $\pi^{L}$ and $\pi^{R}$ are projection on $A$. Since

$$
(\tilde{\varepsilon} \cdot a) \cdot \tilde{\varepsilon}=\tilde{\varepsilon} \cdot(a \cdot \tilde{\varepsilon}),
$$

the projections $\pi^{L}$ and $\pi^{R}$ commute.

Since $\left\|\pi^{L}\right\| \leq 1$ and $\left\|\pi^{R}\right\| \leq 1$, it follows from Tomiyama's theorem [15] that $\pi^{L}$ and $\pi^{R}$ are conditional expectations on $A$.

Since $\pi^{L}$ is a conditional expectation on $A$, for any $a, b \in A$, we have

$$
\tilde{\varepsilon} \cdot((\tilde{\varepsilon} \cdot a) b)=(\tilde{\varepsilon} \cdot a)(\tilde{\varepsilon} \cdot b) \text {. }
$$

Applying $\varepsilon$ to the both sides of this equality we obtain

$$
\tilde{\varepsilon}((\tilde{\varepsilon} \cdot a) b)=\tilde{\varepsilon}(a) \tilde{\varepsilon}(b) .
$$

Let us prove (iii). It suffices to prove (15) for $\xi \geq 0$. Since $\tilde{\varepsilon}$ is a trace on $A, J$ 
coincides with the kernel of the GNS representation constructed from $\tilde{\varepsilon}$. Using the Corollary 3.4.3 in [16] we see that it is sufficient to prove (15) for positive states $\xi$ of the form $\xi(a)=\tilde{\varepsilon}\left(c^{*} a c\right)$, where $a, c \in A$.

Now using (17) and the fact that $\tilde{\varepsilon}$ is a trace we have, for $\xi(a)=\vec{\varepsilon}\left(c^{*} a c\right)$,

$$
\begin{gathered}
\langle\xi \cdot \tilde{\varepsilon}, a\rangle=\langle\xi \otimes \tilde{\varepsilon}, \Delta a\rangle=\xi(\tilde{\varepsilon} \cdot a)=\tilde{\varepsilon}\left(c^{*}(\tilde{\varepsilon} \cdot a) c\right)= \\
=\tilde{\varepsilon}\left(c c^{*}(\tilde{\varepsilon} \cdot a)\right)=\tilde{\varepsilon}\left(c c^{*}\right) \tilde{\varepsilon}(\tilde{\varepsilon} \cdot a)=\tilde{\varepsilon}\left(c c^{*}\right)(\tilde{\varepsilon} \otimes \tilde{\varepsilon}) \Delta a= \\
=\xi(1)\left\langle\tilde{\varepsilon}^{2}, a\right\rangle=\xi(1) \tilde{\varepsilon}(a),
\end{gathered}
$$

whence equality (15) follows. The proof of equality (16) is based on the relation

$$
\tilde{\varepsilon}((a \cdot \tilde{\varepsilon}) b)=\tilde{\varepsilon}(a) \tilde{\varepsilon}(b)
$$

which follows from the fact that $\pi^{R}$ is a conditional expectation.

Let us prove (iv). The embeddings $A / \hat{J} \subset \pi^{R}(A)$ and $\hat{J} \backslash A \subset \pi^{L}(A)$ are obvious. By virtue of (15), for all $\xi \in \hat{J}, a \in A$, we have

$$
\begin{gathered}
\xi \cdot\left(\pi^{L}(a)\right)=\xi \cdot(\tilde{\varepsilon} \cdot a)=(\xi \cdot \tilde{\varepsilon}) \cdot a= \\
=\langle\xi, 1\rangle \tilde{\varepsilon} \cdot a=\langle\xi, 1\rangle \pi^{L}(a),
\end{gathered}
$$

whence the converse embedding $\pi^{L}(A) \subset \hat{J} \backslash A$ follows. The embedding $\pi^{R}(A) \subset A / \hat{J}$ follows from (16).

Theorem 3.1. Let $\mathcal{A}$ be a Kac algebra. Let $P: A \rightarrow B$ be a conditional expectation on the $C^{*}$-algebra $A$ that satisfies all conditions in Theorem 2.1 . Suppose that $\tilde{\varepsilon}=\varepsilon \circ P$ is a tracial state on $A$. Then there exists a Kac algebra $C$ and an epimorphism $\pi: \mathcal{A} \rightarrow C$. Let $P_{1}: A \rightarrow C \backslash A / C$ be the conditional expectation corresponding to the quantum double coset construction. Then there exists a counital conditional expectation $P_{2}: C \backslash A / C \rightarrow B$ such that the diagram

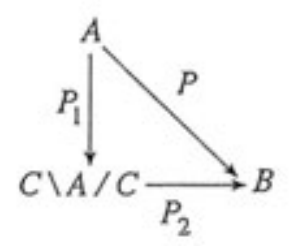

is commutative.

Proof. Denote $P_{1}^{\prime}=\pi^{L} \pi^{R}$. It follows from Lemma 3.5 that $P_{1}^{\prime}$ is a conditional expectation and $P_{1}^{\prime}(A)=\hat{J} \backslash A / \hat{J}$. Since $\hat{J}$ is isomorphic to the dual space of the factor $C^{*}$-algebra $A / C$, the $C^{*}$-algebras $C \backslash A / C$ and $\hat{J} \backslash A / \hat{J}$ coincide.

It follows from the equalities (15), (16) that $\tilde{\varepsilon}$ coincides with the Haar state $\mu_{1}$ on C. Hence

$$
P_{1}(a)=\left(\mu_{1} \circ \pi \otimes \mathrm{id} \otimes \mu_{1} \circ \pi\right) \Delta^{2}(a)=\tilde{\varepsilon} \cdot a \cdot \tilde{\varepsilon}=P_{1}^{\prime}(a),
$$

i. e., $P_{1}^{\prime}$ coincides with the conditional expectation $P_{1}$ that corresponds to the quantum double coset construction.

Denote by $P_{2}=P \uparrow C \backslash A / C$ the restriction of $P$ to the $C$-algebra $C \backslash A / C$. Clearly $P_{2}$ is a conditional expectation that satisfies all conditions in Theorem 2.1 . 
Let us show that conditional expectation $P_{2}$ is counital. For any $a \in C \backslash A / C$ we have

$$
\left(\varepsilon \circ P_{2}\right)(a)=(\varepsilon \circ P)(\tilde{\varepsilon} \cdot a)=\varepsilon(P \otimes \tilde{\varepsilon}) \Delta a=\tilde{\varepsilon}^{2}(a)=\tilde{\varepsilon}(a) .
$$

On the other hand,

$$
\varepsilon(a)=\varepsilon(\tilde{\varepsilon} \cdot a)=(\varepsilon \otimes \tilde{\varepsilon}) \Delta a=\tilde{\varepsilon}(a) .
$$

Hence $\left(\varepsilon \circ P_{2}\right)(a)=\varepsilon(a)$ for all $a \in C \backslash A / C$, i. e., $P_{2}$ is a counital conditional expectation.

Using (6), for any $a \in A$, we have

$$
\begin{gathered}
P\left(P_{1}(a)\right)=P(\tilde{\varepsilon} \cdot a \cdot \tilde{\varepsilon})= \\
=(\varepsilon \otimes \mathrm{id} \otimes \varepsilon)(P \otimes P \otimes P)(\Delta \otimes \mathrm{id}) \Delta a= \\
=(\varepsilon \otimes \mathrm{id} \otimes \varepsilon)(\mathrm{id} \otimes P \otimes \mathrm{id})(\Delta \otimes \mathrm{id})(P \otimes P) \Delta a= \\
=(\varepsilon \otimes \mathrm{id} \otimes \varepsilon)(\mathrm{id} \otimes P \otimes \mathrm{id})(\Delta \otimes \mathrm{id})(P \otimes \mathrm{id}) \Delta P a= \\
=(\varepsilon \otimes \mathrm{id})(\mathrm{id} \otimes P)(P \otimes \varepsilon) \Delta P a= \\
=(\varepsilon \otimes \mathrm{id})(\mathrm{id} \otimes P) \Delta P a=P^{2} a=P a,
\end{gathered}
$$

whence $P=P_{2} P_{1}$, i. e., diagram (18) is commutative.

The authors are deeply grateful to G. B. Podkolzin for many stimulating discussions.

1. Chapoysky Yu. A., Vainerman L. I. Compact quantum hypergroups // J. Operator Theory. -1999. - 41. - P. $261-289$.

2. Berezansky Yu. M., Kalyuzhnyl A. A. Harmonic analysis in hypercomlex systems. - Dordrecht; Boston; London: Kluwer Acad. Publ., 1998. - 483 p.

3. Bloom W. R., Hejer $H$. Harmonic analysis of probability measures on hypergroups. - Berlin; New York: Grueter, 1995. -602 p.

4. Woronowicz S. L. Compact matrix pseudogroups // Communs Math. Phys. - 1987. - 111. P. $613-665$.

5. Chapovsky Yu. A., Vainerman L. $I$. Hypergroup structures associated with a pair of quantum groups $\left(S U_{q}(n), U_{q}(n-1)\right) / /$ Meth. Func. Anal. Probl. Math. Phys. - 1992, - P. $47-69$.

6. Koornwinder T. H. Discrete hypergroups associated with compact quantum Gelfand pairs // Contemp. Math. - 1995. - 183. - P. 213 - 235.

7. Vainerman L. I. Gelfand pairs of quantum groups, hypergroups and $q$-special functions // Ibid. P. $373-394$.

8. Kalyuzhnyi A. A. Conditional expectations on quantum groups and new examples of quantum hypergroups // Meth. Funct. Anal. Top. - 2001. - 7, NN 4, - P. $49-68$.

9. Jewett $R$. Spaces with abstract convolution of mensures // Adv. Math. - 1975. - 18, ND 1. P. $1-101$.

10. Vainerman L. I. 2-Cocycles and twisting of Kac algebras // Communs Math. Phys. - 1998. - 191. -P. $697-721$.

11. Nikshych $D . K_{0}$ rings and twisting of finite-dimensional semisimple Hopf algebras // Communs Algebra. - 1998, - 26, $\mathrm{N}^{0}$ 1. - P. $321-342$.

12. Enock M., Vallin $J .-M . C^{*}$-algebres de Kac et algebres de Kac // Proc. London Math. Soc. 1993. - 66. - P. 610-650.

13. Takesaki $M$. Theory of operator algebras. - New York: Springer, 1979. $-415 \mathrm{p}$.

14. Stratila S. Modular theory in operator algebras. - Kent: Abac. Press, 1967. -512 p.

15. Tomiyama J. On the projection of norm one in $W^{*}$-algebras, I, II, III // Proc. Jap. Acad. Sci. 1957. - 33. - P. 608 - 612; Tohoku Math. J. - 1958. -10. - P. 204-209; 1959. - 11. - P. 125 - 129.

16. Dixmier J. Les $C^{*}$-algebres et leur representations, - Paris: Gauthier-Villars, 1969.

Received 19.12.2002 